\title{
Multiscale correlations and conditional averages in numerical turbulence
}

\author{
Siegfried Grossmann, ${ }^{1, *}$ Detlef Lohse, ${ }^{2, \dagger}$ and Achim Reeh ${ }^{1, \hbar}$ \\ ${ }^{1}$ Fachbereich Physik der Philipps-Universität, Renthof 6, D-35032 Marburg, Germany \\ ${ }^{2}$ Department of Applied Physics and J. M. Burgers Centre for Fluid Dynamics, University of Twente, P.O. Box 217, \\ 7500 AE Enschede, The Netherlands
}

(Received 14 May 1999; revised manuscript received 15 November 1999)

\begin{abstract}
The equations of motion for the $n$th order velocity differences raise the interest in correlation functions containing both large and small scales simultaneously. We consider the scaling of such objects and also their conditional average representation with emphasis on the question of whether they behave differently in the inertial or the viscous subranges. The turbulent flow data are obtained by Navier-Stokes solutions on a $60^{3}$ grid with periodic boundary conditions and $\operatorname{Re}_{\lambda}=70$. Our results complement previous high Re data analysis based on measured data [A. L. Fairhall, V. S. L'vov, and I. Procaccia, Europhys. Lett 43, 277 (1998)] whose preference were the larger scales, and the analysis of both experimental and synthetic turbulence data by [R. Benzi and co-workers, Phys. Rev. Lett. 80, 3244 (1998); Phys. Fluids 11, 2215 (1999)]. The inertial range fusion rule is confirmed and insight is obtained for the conditional averages (the local dissipation rate conditioned on the velocity fluctuations).
\end{abstract}

PACS number(s): 47.27.- $\mathrm{i}$

To analyze the structure of turbulent flow fields Lagrangean longitudinal $n$th order structure functions $S_{n}(R)=\left\langle v^{n}(R)\right\rangle$ are of utmost importance. Here $v(x, R ; t)$ $=[\boldsymbol{u}(\boldsymbol{x}+\boldsymbol{R}, t)-\boldsymbol{u}(\boldsymbol{x}, t)] \cdot \boldsymbol{R} / R$ denotes the longitudinal velocity difference on scale $R$. The equation of motion (cf. [1,2]) in the case of statistically stationary turbulence

$$
\partial_{t} S_{n}(R, t)=0=-n \mathcal{D}_{n}(R, t)+\nu n J_{n}(R, t),
$$

introduces correlation functions of another type, containing local gradient and curvature in addition to the scale $R$. The $(\boldsymbol{u} \cdot \boldsymbol{\nabla}) \boldsymbol{u}$ nonlinearity in the Navier-Stokes equation gives rise to $\mathcal{D}_{n}(R, t)$ (which needs no further specification here because we do not consider it in what follows) and the viscous term $\nu \Delta \boldsymbol{u}$ leads to $J_{n}=\left\langle\Delta u v^{n-1}(R)\right\rangle$. The Laplacean probes the local behavior, while $R$ is in the inertial range. This two-scale character of $J_{n}$ becomes explicit if the local curvature $\Delta u$ is approximated by finite differences, $\Delta_{r} u(x)$ $=[u(x+r)-2 u(x)+u(x-r)] / r^{2}$. The necessity for this discretization arises both in numerical turbulence and in the analysis of measured flow signals. It motivates to study the following more general objects:

$$
\begin{aligned}
J_{n}(r, R)= & r^{-2}\langle[u(x+r)-2 u(x)+u(x-r)] \\
& \left.\times[u(x+R)-u(x)]^{n-1}\right\rangle .
\end{aligned}
$$

Here $r \ll R$ is assumed, and $r$ (instead of $r \rightarrow 0$ ) is allowed to vary in the viscous as well as in the inertial subranges VSR and ISR. In particular $r$ may be below or above $\eta_{n}$, where $\eta_{n}$ denotes the transition scale between VSR and ISR in an nth order correlation function. It is these $J_{n}$ on which we concentrate in this note.

\footnotetext{
*Electronic address: grossmann_s@physik.uni-marburg.de

${ }^{\dagger}$ Electronic address: lohse@tn.utwente.nl

‡Electronic address: reeh@mailer.uni-marburg.de
}

The two-scale correlators $J_{n}$ are of interest to check the validity of the so-called fusion rules $[3,4]$. These describe the proper factorization of the general $n$th order correlation functions

$$
\mathcal{F}_{n}\left(x_{1}, x_{1}^{\prime} ; x_{2}, x_{2}^{\prime} ; \ldots ; x_{n}, x_{n}^{\prime}\right):=\left\langle v\left(x_{1}, x_{1}^{\prime}\right) \cdots v\left(x_{n}, x_{n}^{\prime}\right)\right\rangle,
$$

if a subgroup of $p$ pairs $\boldsymbol{x}_{i}-\boldsymbol{x}_{i}^{\prime}$ becomes much smaller, "fuses," than the remaining $n-p$ pairs, $1<p<n$. Let the fusing pairs have scale $r$, the nonfusing ones scale $R$. If $r$ $\ll R$, the $r$ eddies are supposed to be statistically independent from the large scale motion $R$ and vice versa. This might naively suggest to factorize $\mathcal{F}_{n}(r, R) \approx \mathcal{F}_{p}(r) \mathcal{F}_{n-p}(R)$. But this turns out as too naive, because it contradicts the scaling of the $n$th order correlation functions under dilation of distances, unless the scaling exponents $\zeta_{n}$ are linear in $n$. Namely, define the scaling exponents $\zeta_{n}$ by

$$
\mathcal{F}_{n}(\lambda r, \lambda R)=\lambda^{\zeta_{n}} \mathcal{F}_{n}(r, R)
$$

The naive factorization immediately leads to $\zeta_{n}=\zeta_{p}$ $+\zeta_{n-p}$. Thus a relation between the scaling exponents obtains which implies $\zeta_{n}=\zeta_{1} n$, meaning that only one nontrivial scaling exponent exists ("monoscaling"). To allow for the possibility of multiscaling, equivalent to nonlinear $n$ dependence of the scaling exponents, the naive factorization ansatz has to be avoided, because it jeopardizes multiscaling by assumption.

A more general factorization of the multiscale correlation functions $\mathcal{F}_{n}(r, R)$, denoted as 'fusion rule"' by L'vov and Procaccia [3,4], starts with the decomposition $\mathcal{F}_{n}(r, R)$ $=\mathcal{F}_{p}(r) \widetilde{\mathcal{F}}_{p, n-p}(r, R)$, the second factor being defined by this equation. Here it is assumed that the small $r$ eddies move in the field of the larger, slower $R$ eddies as they would do also without the large scale motion, and are described therefore by the $p$ correlator of the $p$ smaller $r$ eddies $v_{i}(r)$. The statistical independence of the small and the large scales is expressed by skipping the $r$ dependence of the large scale eddy 
correlation $\widetilde{\mathcal{F}}_{p, n-p}(r, R)$. But even if $\widetilde{\mathcal{F}}_{p, n-p}(R)$ is assumed to be independent of the small scale $r$, it may remember its origin from an $n$-point correlator. Performing the dilation transformation of the product $\mathcal{F}_{p} \widetilde{\mathcal{F}}_{p, n-p}$ of correlation functions with $\lambda$ leads to

$$
\widetilde{\mathcal{F}}_{p, n-p}(\lambda R)=\lambda^{\zeta_{n}-\zeta_{p}} \widetilde{\mathcal{F}}_{p, n-p}(R),
$$

provided $r$ and $R$ are in the same, inertial scaling range. The scaling behavior (4) is obtained if $\widetilde{\mathcal{F}}_{p, n-p}(R)$ is expressed as the ratio of an $n$th and a $p$ th order correlation function, i.e., $\widetilde{\mathcal{F}}_{p, n-p}(R) \propto S_{n}(R) / S_{p}(R)$, and therefore the scaling invariant factorization reads

$$
\mathcal{F}_{n}(r, R) \sim S_{p}(r) S_{n}(R) / S_{p}(R) .
$$

The symbol $\sim$ is to be understood as "scaling wise." Clearly both the left-hand side (LHS) and the RHS rescale under $\lambda$ as $\lambda^{\zeta_{n}}$, thus avoiding any prejudice in the factorization about multiscaling or not multiscaling.

An alternative derivation of Eq. (5) is given in Ref. [7], based on the multifractal view of the turbulent cascade. It does not explicitly make use of the Navier-Stokes equation. That approach can even account for higher order corrections to the prediction (5), see below.

The importance of verifying the factorization ("fusion rule') (5) is evident. We do this here by applying it to the $J_{n}(r, R)$ of Eq. (2). Decompose at first $v^{n-1}(R)$ in Eq. (2) into $[u(x+R)-u(x)] v^{n-2}(R)$. Then, next, respect $u(x$ $\pm r) u(x+R)$ and thus also $u(x) u(x+R)$ to have correlation 0 as the consequence of the statistical independence of the velocities at two widely separated positions $\boldsymbol{x}+\boldsymbol{r}, \boldsymbol{x}+\boldsymbol{R}$ because $r \ll R$. These correlations are even supposed to vanish if the additional factor $v^{n-2}(R)$ is included. Thus we are left with

$$
J_{n}(r, R)=r^{-2}\left\langle\left[2 u^{2}(x)-u(x+r) u(x)-u(x-r) u(x)\right] v^{n-2}(R)\right\rangle .
$$

Because of spatial translational invariance this can be rearranged to give

$$
J_{n}(r, R)=r^{-2}\left\langle v^{2}(r) v^{n-2}(R)\right\rangle, r \ll R .
$$

The objects $J_{n}$ are now in the form of a two-scale correlation function to which the factorization ("fusion") rules can be applied and their validity be checked. We do this for $r$ in the ISR according to Eq. (5) and later on also for $r$ in the VSR.

Let us first consider the fusion rule for $\eta_{n} \ll r \ll R \ll L$, both scales being in the ISR ( $L$ being the outer scale). It says by Eq. (5) that

$$
J_{n}(r, R)=C_{n} r^{-2} S_{2}(r) S_{n}(R) / S_{2}(R)
$$

All these correlation functions $S_{n}$ and $S_{2}$ can easily be computed from a numerical turbulent flow solution of the NavierStokes equation, the $J_{n}(r, R)$ according to their defining formula (2), and the $S_{n}$ according to their definition $\left\langle v^{n}\right\rangle$. If $n$ is odd it makes a difference if $v^{n}$ or $|v|^{n}=\left(\sqrt{v^{2}}\right)^{n}$ is used, see Ref. [5]; we distinguish these cases as $S_{n}$ and $S_{n}^{*}$. Typical averaging times are about 100 large eddy turnovers. Forcing is on the largest scale. The periodicity length of the box then is $2 \pi L$. Lengths are nondimensionalized with this length $L$. Time is nondimensionalized with $\left(L^{2} / \epsilon\right)^{1 / 3}$. Thus the nondimensional energy dissipation rate per mass becomes $\epsilon=1$. The (dimensionless) velocities are always divided by the rms velocity, which in our flow turns out to be $u_{\mathrm{rms}}=1.40$. The viscous length scale for the second order correlations is $\eta_{2}$ $\equiv \eta=\left(\nu^{3} / \epsilon\right)^{1 / 4}=9 \times 10^{-3} L$. For the averaging the vectors $\boldsymbol{r}$ and $\boldsymbol{R}$ are chosen as $r(1,0,0)$ and $R(1,0,0)$. The isotropy of the flow has carefully been checked. For more details see Ref. [6].

To concentrate on the dependence on one variable scale, we calculated the ratios

$$
J_{n}(r, R) / J_{2}(r, R)=\left(C_{n} / C_{2}\right) S_{n}(R) / S_{2}(R),
$$

displayed in Figs. 1 and 2 for $n=4$ and 6, respectively. For these plots we have divided the LHS of Eq. (8) by the expected scaling behavior $S_{n}(R) / S_{2}(R)$. Therefore, if Eq. (8) holds, one would have a straight line. Indeed, for the ISR scales $r=18 \eta$ and $r=36 \eta$ where Eq. (8) is supposed to hold, we see such behavior, see the upper curves in Figs. 1 and 2.

The derivation of Benzi et al. [7] of the fusion rules, which is only based on the assumption of an uncorrelated multiplicative process for the energy cascade, is able to even give the correction term in Eq. (8). We will show this explic-

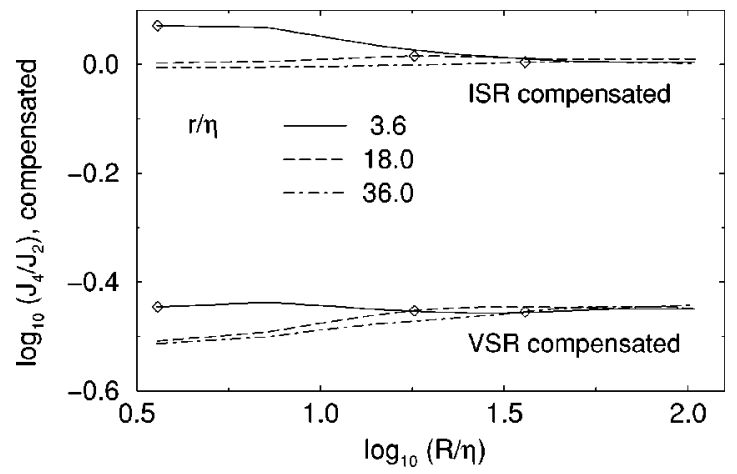

FIG. 1. The upper curves are (in a double logarithmic plot) $J_{4}(r, R) J_{2}^{-1}(r, R) / S_{4}(R) S_{2}^{-1}(R)$ versus $R / \eta$. Here $\eta$ is $\left(\nu^{3} / \epsilon\right)^{1 / 4}$ and $\epsilon=1$ in our units. From top to bottom $r / \eta=3.6,18$, and 36, thus from VSR to ISR. The symbols $\diamond$ indicate that here $r=R$, i.e., only at the right of $\diamond$ the assumption $r \ll R$ can be considered as (at least approximately) fulfilled. The lower triple of curves shows the analogous information if $J_{4}(r, R) J_{2}^{-1}(r, R) / S_{5}(R) S_{3}^{-1}(R)$ is plotted on the ordinate, i.e., compensation with the VSR fusion rule (15) is chosen. The inertial range starts at $R / \eta=\mathcal{O}(10)$. 


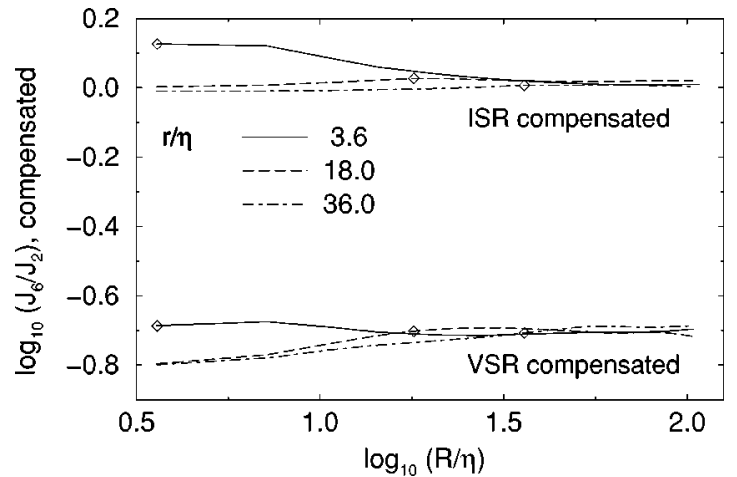

FIG. 2. The corresponding results for $n=6$ as in Fig. 1 for the case $n=4 . J_{6}(r, R) J_{2}^{-1}(r, R)$ is divided by the ISR fusion rule prediction $S_{6}(R) S_{2}^{-1}(R)$ in the upper triple of curves and by the VSR fusion rule prediction $S_{7}(R) S_{3}^{-1}(R)$ in the lower triple. Again $r$ $<R$ holds on the right of the symbols $\diamond$. The VSR-ISR transition is at $R / \eta=\mathcal{O}(10)$.

itly for the case of $J_{4}(r, R)$ : Following Ref. [7] and applying the so called "Ward identity," we first get the exact relation

$$
\begin{aligned}
S_{4}(R-r)= & S_{4}(R)+S_{4}(r)-4 \widetilde{\mathcal{F}}_{1,3}(r, R)-4 \widetilde{\mathcal{F}}_{3,1}(r, R) \\
& +6 \widetilde{\mathcal{F}}_{2,2}(r, R) .
\end{aligned}
$$

In the limit of $r / R \rightarrow 0$ such that $S_{4}(R-r) \approx S_{4}(R)(1$ $-\zeta_{r} r / R$ ) and with Eq. (10) of Ref. [7] for $\widetilde{\mathcal{F}}_{3,1}=\widetilde{\mathcal{F}}_{1,3}$ (for which the fusion rules erroneously give 0 as elaborated in Ref. [7]) we finally obtain the higher order correction in Eq. (8), namely,

$$
\begin{aligned}
\frac{J_{4}(r, R)}{J_{2}(r, R)}= & \frac{C_{4}}{C_{2}} \frac{S_{4}(R)}{S_{2}(R)}\left[1+O\left(\frac{r S_{2}(R)}{R S_{2}(r)}\right)+O\left(\frac{S_{3}(r) S_{2}(R)}{S_{3}(R) S_{2}(r)}\right)\right. \\
& \left.+O\left(\frac{S_{4}(r) S_{2}(R)}{S_{4}(R) S_{2}(r)}\right)\right]
\end{aligned}
$$

The first two correction terms on the RHS are-up to intermittency corrections - order of $(r / R)^{1 / 3}$, the last one is order of $(r / R)^{2 / 3}$. Indeed, qualitatively we see such a correction in Fig. 1, upper, for the $r / \eta=3.6$ curve: The smaller $R$, the larger the correction to the plateau. [For the other two curves the condition $r / R \ll 1$ used in the derivation of Eq. (10) is not fulfilled throughout.] Unfortunately, the scaling exponents of the correction terms cannot be extracted due to the short scaling regime.

In order to judge whether the horizontal lines in Figs. 1 and 2 are incidental, we have studied in addition another plausible, alternative factorization formula that might stand instead of Eq. (5). Apparently, $r$ and $R$ have not been treated symmetrically, when factorizing $\mathcal{F}_{n}(r, R)$. If one argues differently than before that the $n-p$ larger eddies, because they carry the energy, are correlated as if the smaller $r$ eddies were not present, the decomposition $\mathcal{F}_{n}(r, R)$ $=\mathcal{F}_{n-p}(R) \check{\mathcal{F}}_{p, n}(r, R)$ would be natural. As before, statistical independence of the $r$ and $R$ eddies lets one skip the $R$ dependence in $\check{\mathcal{F}}_{p, n}$. The conservation of the global scaling $\propto \lambda \zeta_{n}$ then leads to

$$
\check{\mathcal{F}}_{p, n}(\lambda r)=\lambda^{\zeta_{n}-\zeta_{n-p}} \check{\mathcal{F}}_{p, n}(r)
$$

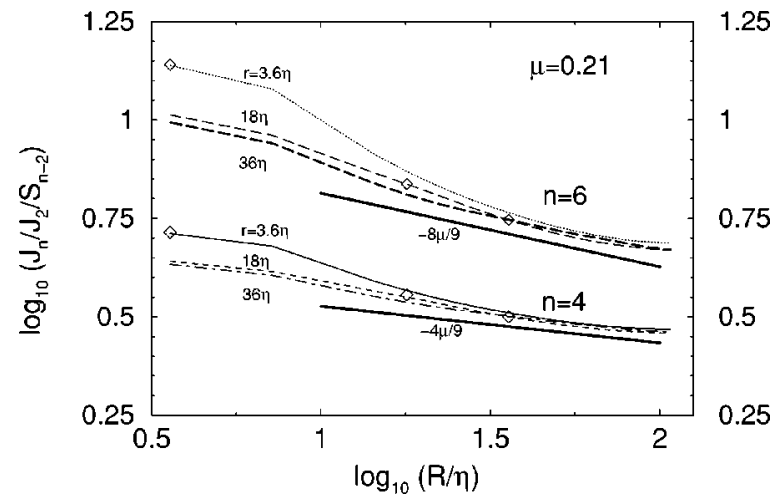

FIG. 3. Compensated plots $J_{n} J_{2}^{-1} / S_{n-2}$ as functions of $R / \eta$ for $n=4$ (lower) and $n=6$ (upper), choosing $r=3.6 \eta, 18 \eta$, and $36 \eta$. Again $\diamond$ indicates where $R=r$; the relevant range $r<R$ is towards the right. The full straight lines represent the slopes $-4 \mu / 9$ $=-0.093$ and $-8 \mu / 9=-0.187$ for $\mu=0.21$ (taken from [6] as an appropriate fit value in the K62 intermittency model). $J_{n} / J_{2}$ clearly seems to behave as $S_{n}(R) / S_{2}(R)$ instead of $S_{n-2}(R)$.

As a consequence scaling-wise we have $\check{\mathcal{F}}_{p, n}(r)$ $\propto S_{n}(r) / S_{n-p}(r)$, altogether the alternative fusion rule

$$
\mathcal{F}_{n}(r, R) \sim S_{n-p}(R) S_{n}(r) / S_{n-p}(r) .
$$

In contrast to the original factorization $\sim R^{\zeta_{n}-\zeta_{p}}$ this one scales as $R^{\zeta_{n-p}}$, in both cases the global $R$ and $r$ scaling being conserved, $\propto \lambda^{\zeta_{n}}$. There is no difference in case of monoscaling, while for multiscaling, $\zeta_{n-p} \neq \zeta_{n}-\zeta_{p}$, both decompositions differ. (7),

Applying Eq. (12) to $J_{n}$ of Eq. (6) we find instead of Eq.

$$
J_{n}(r, R) \propto r^{-2} S_{n}(r) S_{n-2}^{-1}(r) S_{n-2}(R) .
$$

Accordingly

$$
J_{n}(r, R) / J_{2}(r, R)=\hat{C}_{n}(r) S_{n-2}(R),
$$

with $\hat{C}_{n}(r) \propto S_{n}(r) /\left[S_{2}(r) S_{n-2}(r)\right]$, since $S_{0}(R)=S_{0}(r)=1$. Figure 3 presents compensated plots according to this alternative ISR fusion rule. Apparently, the original fusion rules Eq. (5) and (8), respectively, are superior, the alternatives (12) and (14) can be discarded.

One can understand the different slopes in Fig. 3 relative to Figs. 1,2 (upper) even quantitatively. The difference of the slopes for the two ISR fusion rules is $\delta_{n}=\zeta_{n}-\zeta_{2}-\zeta_{n-2}$. It fully indicates multiscaling, because $\delta_{n}=0$ in the monoscaling case K41. For simplicity we consider the multiscaling model K62 (see Refs. [8,9]) $\zeta_{n}=n / 3-\mu n(n-3) / 18$ and find $\delta_{n}=-(2 \mu / 9)(n-2)$. In particular $\delta_{2}=0, \delta_{4}=-4 \mu / 9$, and $\delta_{6}=-8 \mu / 9$. These slopes are marked in Fig. 3 and seem to be consistent with the empirical slopes in the compensated curves of the alternative fusion rule. Given the small Reynolds number, one can of course argue whether there is scaling at all.

We conclude that the scaling of the $n-p$ large $R$ eddies is well affected by the presence of the $p$ smaller $r$ eddies, this effect being due completely to multiscaling. In contrast, the small $r$ eddies are curling as if they were free, uneffected by the large eddies in whose field they move. 
The factorization rule (7) for the choice $\eta_{n} \ll r \ll R$ has been confirmed previously already with measured data [10]. [In Ref. [10] the $J_{n}$ erroneously have an additional factor $n / 2$; for the structure functions the $S_{n}^{*}(R)$ are used.] Due to restrictions in the small $r$ resolution of the measured data, a verification of the VSR fusion rules was not possible. This was another motivation for the present numerical work.

We now consider the easily accessible case when $r$ is in the VSR, i.e., $r / \eta<\mathcal{O}(10)$. The argument for the factorization (5) was based on strictly keeping the scaling exponent $\zeta_{n}$ of $\mathcal{F}_{n}$ also in its factorized form. We tacitly assumed that the small $r$ as well as the large $R$ scaling exponents are the ISR type $\zeta_{p}$, which is natural if both small $r$ and large $R$ are in the ISR. If $r \in \mathrm{VSR}$, its scaling exponent $\zeta_{p}$ is regular, instead, $\zeta_{p} \rightarrow p$. Then the homogeneity exponent of $\widetilde{\mathcal{F}}_{p, n-p}(R)$ under rescaling with $\lambda$ is $\zeta_{n-p}$ instead of $\zeta_{n}-\zeta_{p}$. Therefore $\widetilde{\mathcal{F}}_{p, n-p}(R)$ can no longer be described by the ratio $S_{n}(R) / S_{p}(R)$ because $R$ is in the ISR and thus the denominator has the scaling behavior $\zeta_{p}$, but not $p$ as desired. Therefore the ISR factorization rule (7) is expected to become invalid if $r$ is below $\approx 10 \eta$. This is supported by Figs. 1 and 2 (lower).

Incidentally, if both $r$ and $R$ are in the VSR they both have regular monoscaling exponents $p$ and $n-p$. But here the argument of statistical independence due to $r \ll R$ ceases to be valid, i.e., no factorization is possible anymore; and it is not necessary either, because there is regular $r, R$ dependence.

If $r \ll \eta_{n} \ll R \ll L$, denoted as the ( $r$ ) VSR case, two competing factorizations are at hand. One of them is derived in Refs. [2] and [1], saying

$$
J_{n}(r, R)=\widetilde{C}_{n} S_{n+1}(R) / S_{3}(R) .
$$

The other one, which strictly adheres to the invariance of the total $\mathcal{F}_{n}$ scaling behavior under factorization reads

$$
\begin{gathered}
J_{n}(r, R)=\hat{C}_{n} \frac{S_{2}(r)}{r^{2}} \frac{S_{n}(R)}{R^{2}} \sim \frac{S_{2}(r)}{r^{2}} \frac{S_{n}(R)}{S_{3}^{2}(R)}, \\
r \in \mathrm{VSR}, R \in \mathrm{ISR}, r \ll R .
\end{gathered}
$$

This factorization is based on the assumption that the $n$th order correlation has the homogeneity exponent $\zeta_{n}$ irrespective of $r, R$ being in the same or in different subranges. The RHS has scaling exponent $\zeta_{n}$, because $S_{2}(\lambda r) \propto \lambda^{2} S_{2}(r)$ for $r \in$ VSR and also $S_{3}^{2}(\lambda R)=\lambda^{2} S_{3}^{2}(R)$ for $R \in$ ISR, since $S_{3}$ $\propto R$ from the Howard-van Kármán-Kolmogorov structure equation. [More generally $\mathcal{F}_{n}(r, R) \propto S_{p}(r) S_{n}(R) / S_{3}^{p}(R)$.]

There is not much difference between Eqs. (15) and (16) in the small $r$ dependence, because $S_{2}(r) \propto r^{2}$ for small $r$. The factorization (15) says that the LHS is independent of $r$ once $r$ is less than $\eta$. It furthermore states that the $n-2$ factors of $v(R)$ in $J_{n}$ scale with exponent $\zeta_{n+1}-1$ or, if

$$
J_{n}(\lambda r, \lambda R)=\lambda^{\tilde{\zeta}_{n}} J_{n}(r, R) \text { then } \widetilde{\zeta}_{n}=\zeta_{n+1}-1 .
$$

Since in the equation of motion the $r \rightarrow 0$ limit of $J_{n}(r, R)$ is needed, the VSR factorization ("fusion") rule is of considerable importance. The quality of Eq. (15) for numerical turbulence is tested in Figs. 1 and 2, lower triples respectively.

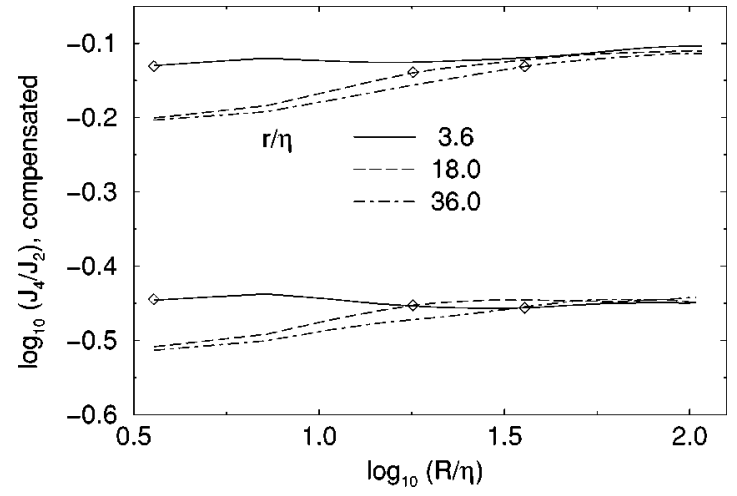

FIG. 4. The VSR factorization (15), i.e., $J_{4} J_{2}^{-1}$ divided by $S_{5}^{*} S_{3}^{*-1}$ (upper triple) or by $S_{5} S_{3}^{-1}$ (lower triple). The latter is the same as in Fig. 1. For more information see the caption of Fig. 1. While the two triples of curves are structurally similar, there are differences in detail if the $S_{n}^{*}$ or the $S_{n}$ are taken.

Again, we use compensated plots, this time dividing $J_{n} / J_{2}$ by the expected VSR-scaling behavior, i.e., the right hand side of Eq. (15). If that holds, one would again expect a straight line. Indeed, if $r=3.6 \eta$, i.e., $r$ is in the VSR, the line is straighter than for $r$ values in the ISR, but the result is not completely conclusive because of the low Reynolds number.

We also present Figs. 4 and 5 in order to identify possible differences if $S_{n}^{*}$ is taken instead of $S_{n}$. This is irrelevant in the case of the inertial range factorization rule, since the involved $n=2,4,6$ are all even. But the VSR factorization (15) comprises odd $n$. As Figs. 4 and 5 show there are noticable differences in the details of the respective upper and lower triples, but the curves are structurally the same.

An argument leading to Eq. (15) is start with the ISR fusion rule (7) and reduce $r$ continuously to submerge in the VSR eventually. That means, keep the $R$ dependence of the RHS and fix $r$ at the crossover from the ISR to the VSR, i.e., fix $r$ at $\mathcal{O}\left(\eta_{n}\right)$. Then $r^{-2} S_{2}(r) \propto \eta_{n}^{-2} \eta_{n}^{\zeta_{2}}$. Now it is important that the crossover scale $\eta_{n}$ not only depends on the order $n$ of the moment but also on the scale $R$, see [1], according to

$$
\eta_{n}=\eta(R / L)^{x_{n}},
$$

with

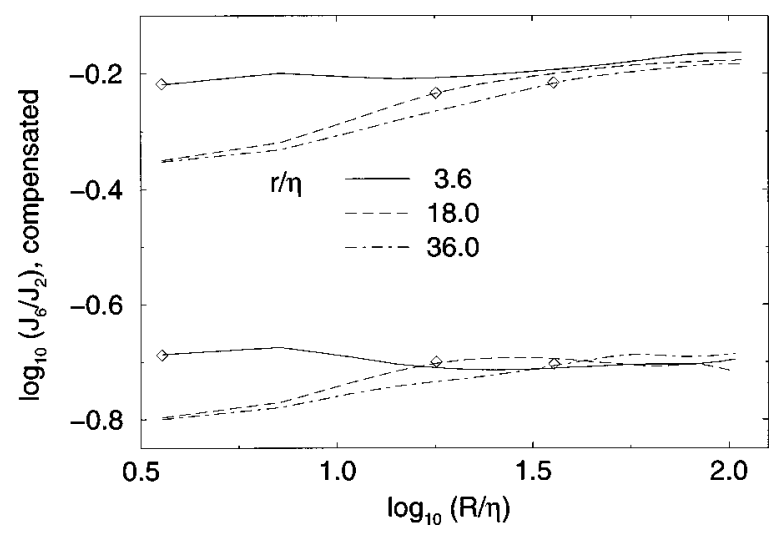

FIG. 5. The VSR factorization (15) of $J_{6} J_{2}^{-1}$ divided by $S_{7}^{*} S_{3}^{*-1}$ (upper triple) or by $S_{7} S_{3}^{-1}$ (lower triple). Again there are differences in the detailed behaviors between upper and lower. 


$$
x_{n}=\left(\zeta_{n}+\zeta_{3}-\zeta_{n+1}-\zeta_{2}\right) /\left(2-\zeta_{2}\right)
$$

In particular, $\eta_{2}=\eta$ as it should. The $R$ dependence of the $\eta_{n}$ as well as their $n$ dependence originates from multiscaling as the signature of intermittency. In K41 one has $x_{n}=0$ and $\eta_{n}=\eta$ for all $n$. Relation (18) implies

$$
\left.r^{2} S_{2}(r)\right|_{r \approx \eta_{n}} \propto R^{\zeta_{n+1}+\zeta_{2}-\zeta_{n}-\zeta_{3},}
$$

which when used together with Eq. (7) leads to

$$
J_{n}(r \approx \eta, R) \propto R^{\zeta_{n+1}-\zeta_{3}} .
$$

This is just Eq. (15). For monoscaling (linear $n$ dependence) this coincides with Eq. (7) at $r=\eta$. It therefore is of high interest to identify the validity and the differences between Eqs. (7) and (15), the fusion rules in the $r$-ISR and $r$-VSR cases, with convincing significance.

Both $r$-VSR factorization rules (15) and (16) might not be considered as based on safe arguments. It therefore seems adequate to study the special case $n=2$ separately, where everything can be evaluated explicitly. Only the statistical spatial homogeneity of the flow is used. Start with Eq. (2), choose $n=2$ and find $r^{2} J_{2}(r, R)=\langle u(\boldsymbol{x}+\boldsymbol{r}) u(\boldsymbol{x}+\boldsymbol{R})$ $-2 u(\boldsymbol{x}) u(\boldsymbol{x}+\boldsymbol{R})+u(\boldsymbol{x}-\boldsymbol{r}) u(\boldsymbol{x}+\boldsymbol{R})-u(\boldsymbol{x}+\boldsymbol{r}) u(\boldsymbol{x})+2 u^{2}(\boldsymbol{x})$ $-u(\boldsymbol{x}-\boldsymbol{r}) u(\boldsymbol{x})\rangle$. Employing translational invariance, e.g., $u(\boldsymbol{x}+\boldsymbol{r}) u(\boldsymbol{x}+\boldsymbol{R}) \hat{=} u(\boldsymbol{x}) u(\boldsymbol{x}+\boldsymbol{R}-\boldsymbol{r})$, etc., straightforwardly leads to the relation

$$
\begin{aligned}
J_{2}(r, R)= & r^{-2}\left\{S_{2}(r)+\left[S_{2}(R)-\frac{1}{2}\left(S_{2}(R+r)\right.\right.\right. \\
& \left.\left.\left.+S_{2}(R-r)\right)\right]\right\} .
\end{aligned}
$$

In the limit $r \rightarrow 0$ one can expand $S_{2}(r)$ and $[\cdots]$ up to second order in $r$ and finds

$$
J_{2}(r, R)=\left\langle\left(\partial_{1} u_{1}\right)^{2}\right\rangle-\frac{1}{2} \frac{d^{2} S_{2}}{d R^{2}} .
$$

If $R$ is in the ISR, the second term becomes small as $R^{\zeta_{2}-2}$, i.e., roughly $\propto R^{-4 / 3}$, and the first, constant term remains. This is compatible with Eq. (15) for $n=2$, if $\hat{C}_{2}$ $=\left\langle\left(\partial_{1} u_{1}\right)^{2}\right\rangle$, while Eq. (16) is excluded because it approaches 0 as $\propto R^{-4 / 3}$. Also the ISR fusion rule (7) for $n$ $=2$ is reproduced to leading order. On the other hand, the functional dependence of $J_{2}(r, R)$ on both $r$ and $R$ does not change if $r$ passes from the ISR to the VSR. The only relevant property is $r \ll R$.

A generalization of Eq. (22) for $n>2$ has been derived in Ref. [7].

$$
J_{n}(r \rightarrow 0, R)=\hat{C}_{n}\left\langle\left(\partial_{1} u_{1}\right)^{2}\right\rangle \frac{S_{n}(R)}{S_{2}(R)}-\frac{1}{n} \frac{d^{2}}{d R^{2}} S_{n}(R), R \in \text { ISR. }
$$

If $n$ is large enough, the second term does no longer decrease with increasing $R$. It grows instead. But still its size shrinks relative to the first term as $R^{\zeta_{2}-2} \rightarrow 0$. For $n=2$ Eq. (22) is recovered. Note that the generalization (23), for large enough
$R$ to neglect the second term, well reproduces the $r$-ISR fusion rule (7) but does not coincide with the $r$-VSR rule (15) as it should, since $r \rightarrow 0$ is considered.

To analyze the differences between the ISR and the VSR factorization rules further we now consider the average (discretized) curvature $\Delta_{r} u$ under the condition that the $R$-eddy velocity difference $v(x, R ; t)$ has the value $v_{R}$. We denote this conditional average as $\left\langle\Delta_{r} u \mid v_{R}\right\rangle$. It allows us to compute the $J_{n}(r, R)$ from (2) by using the $v_{R}$-probability densities,

$$
J_{n}(r, R)=\int\left\langle\Delta_{r} u \mid v_{R}\right\rangle v_{R}^{n-1} P_{R}\left(v_{R}\right) d v_{R} .
$$

Here $P_{R}\left(v_{R}\right)$ is the (unconditioned) probability density to find the $R$-eddy velocity difference $v_{R}$, which can be obtained directly from the numerical solution for each chosen eddy size $R$, on which $P_{R}$ depends.

The conditional averages $\left\langle\Delta_{r} u \mid v_{R}\right\rangle$ can be calculated via unconditioned probabilities. It is

$$
\left\langle\Delta_{r} u \mid v_{R}\right\rangle=\int P_{r, R}\left(\kappa \mid v_{R}\right) \kappa d \kappa=\int \frac{P_{r, R}\left(\kappa, v_{R}\right)}{P_{R}\left(v_{R}\right)} \kappa d \kappa .
$$

Here $P_{r, R}\left(\kappa, v_{R}\right)$ is the common joint probability density to find the field curvature $\kappa \hat{=} \Delta_{r} u$ and the $R$-eddy velocity $v_{R}$. The fraction has the meaning of the conditional probability density $P_{r, R}\left(\kappa \mid v_{R}\right)$.

The fusion rules (7) and (15) follow immediately if the conditional curvature averages have linear or quadratic dependence on the conditioning eddy field $v_{R}$, as was indicated in Refs. [1,10]. In the $r$-ISR case $\eta \ll r \ll R$ the linear ansatz

$$
\begin{aligned}
\left\langle\Delta_{r} u \mid v_{R}\right\rangle & =\left[C_{n} r^{-2} S_{2}(r) / S_{2}(R)\right] v_{R} \\
& =\left[J_{2}(r, R) / S_{2}(R)\right] v_{R}
\end{aligned}
$$

inserted into Eq. (24) immediately leads to Eq. (7). The quadratic $v_{R}$ dependence

$$
\left\langle\Delta_{r} u \mid v_{R}\right\rangle=\left[\widetilde{C}_{n} / S_{3}(R)\right] v_{R}^{2}
$$

in turn gives the $r$-VSR factorization (15). To be more precise, the ansatz (27) is a sufficient condition to imply Eq. (15). Alternatively, as argued in Ref. [11], $\left\langle\Delta_{r} u \mid v_{R}\right\rangle$ may be an infinite series in $v_{R}$, of which we shall study the beginning, see Eq. (28). The dependencies (26) and (27) on $v_{R}$ are so markedly different that the conditional average representation (24) seems qualified to become a sensitive check. If Eqs. (26) and (27) could be proven to hold, the validity of the fusion rules (7) and (15) in the $r$-ISR and the $r$-VSR cases were strongly supported.

We have determined the conditional averages $\left\langle\Delta_{r} u \mid v_{R}\right\rangle$ numerically via $P_{r, R}\left(\kappa, v_{R}\right)$ and $P_{R}\left(v_{R}\right)$ from Eq. (25) for two values of $r$, one in the ISR, $r=36 \eta$, the other one in the VSR, $r=3.6 \eta$, as functions of $v_{R}$ for various fixed $R$. The curves are displayed in Figs. 6 and 7.

While the linear dependence of $\left\langle\Delta_{r} u \mid v_{R}\right\rangle$ nicely confirms the ISR fusion rule (7), the completely missing quadratic $v_{R}$ dependence (27) excludes the possibility to understand Eq. (15) from a simple product ansatz for the conditional prob- 


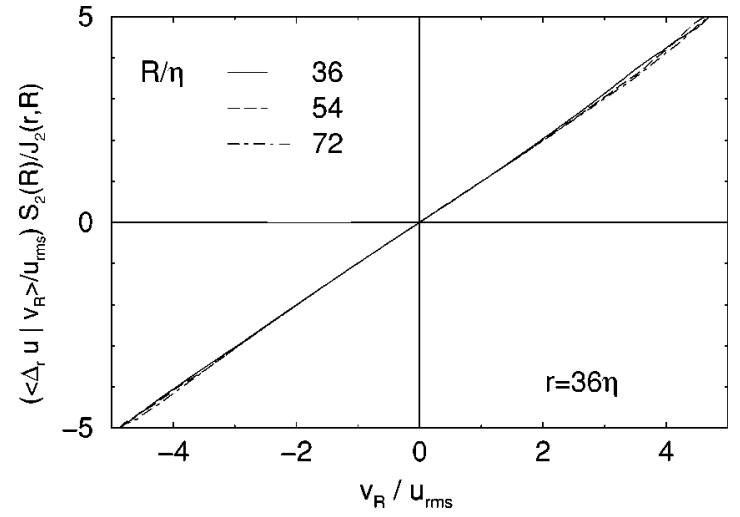

FIG. 6. The conditional average $\left\langle\Delta_{r} u \mid v_{R}\right\rangle$ in multiples of $J_{2}(r, R) / S_{2}(R)$ versus $v_{R}$ in multiples of $u_{\text {rms }}(=1.40)$ in a double logarithmic plot. $r=36 \eta$ is in the ISR. The large eddy scale $R$ varies through $36 \eta, 54 \eta, 72 \eta$, all in the ISR or even in the stirring subrange. The expected linear behavior (26) is evident. The slope of the linear part is 1 , as it should.

ability $\left\langle\Delta_{r} u \mid v_{R}\right\rangle$, leaving the possibility of an infinite series expansion as stated in Ref. [11] to be tested. The alternative (16), on the other hand, is consistent, $J_{n} \propto S_{n}(R)$. Linear laws for the conditional averages have been discussed also in the context of passive scalar advection [11-15]. For the choice $r \in$ ISR this linear $v_{R}$ dependence has been studied for Navier-Stokes turbulence by Fairhall et al. [10]. In the present paper we add data on $r$-VSR conditional averages.

We can check the direct method (2) to calculate the $J_{n}$ and the conditional average representation (24) against each other, see Figs. 8 and 9 for the cases $n=2$ and 3. We have also evaluated $J_{4}$ and $J_{6}$ and have found similar agreement between the various methods [Eqs. (2) or (24)] as in the case $J_{2}$. The corresponding curves are not displayed here.

To a good approximation the conditional average of the field curvature $\Delta_{r} u$ depends linearly on $v_{R}$, irrespective of $r$ being in the ISR or in the VSR, above or below $\eta$. This agrees nicely with the observation in Eq. (21) that $\eta$ is no explicitly relevant quantity for $J_{2}(r, R)$.

To control the deviations from the linear behavior we fit-

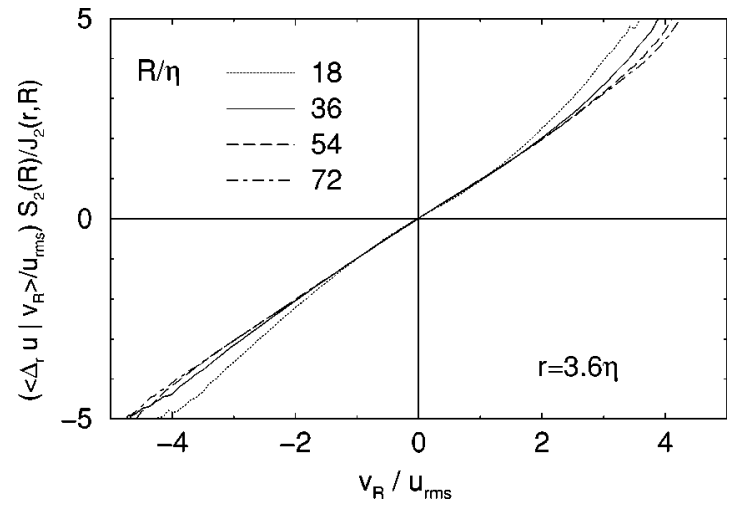

FIG. 7. The conditional average $\left\langle\Delta_{r} u \mid v_{R}\right\rangle$ if $r=3.6 \eta$ is in the VSR; the same values of $R$ as in Fig. 5. With convincing evidence it is not quadratic with respect to $v_{R}$ but linear, too, at least for negative $v_{R}$ and up to $\approx 3 u_{\text {rms }}$ for positive ones. $u_{\text {rms }}$, by the way, is 1.40 . We believe, this asymmetry is a consequence of insufficient statistics for the large fluctuations $\left|v_{R}\right|$ and has not necessarily significance.
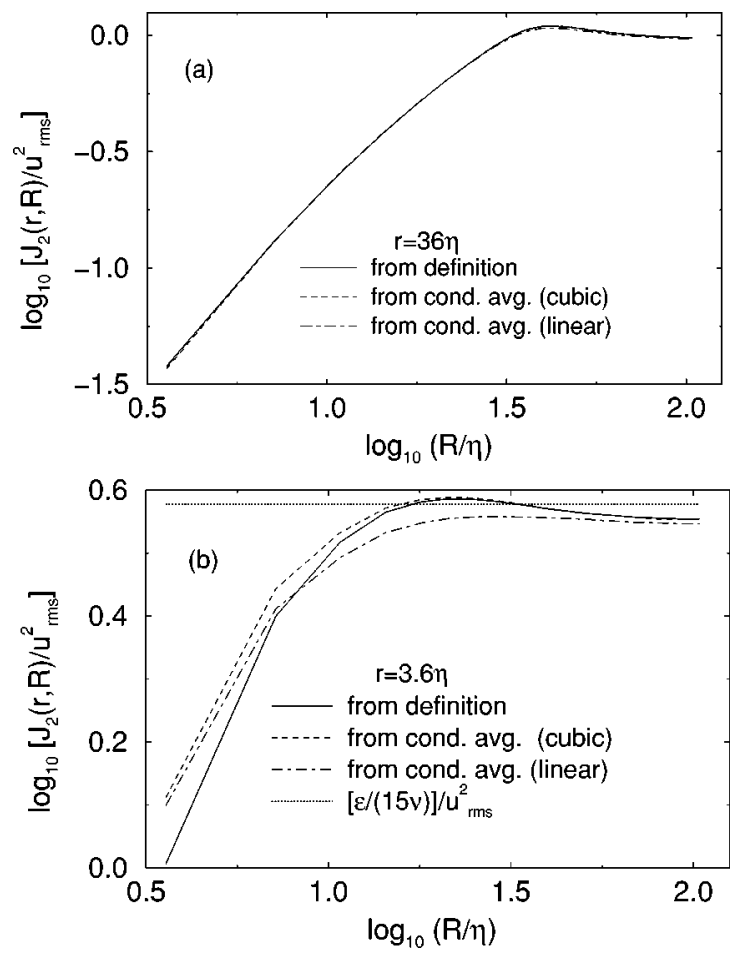

FIG. 8. (a) Double logarithmic plot of $J_{2}(r, R)$ versus $R / \eta$ for $r=36 \eta$ in the ISR. The directly calculated $J_{2}$ from the definition (2) and that from the conditional average representation (24) very well agree, for both the linear and the linear plus cubic interpolation (28) of $\left\langle\Delta_{r} u \mid v_{R}\right\rangle$. (b) $J_{2}(r, R)$ versus $R / \eta$ if $r=3.6 \eta$ is in the VSR. We can compare with the constant value of $\left\langle\left(\partial_{1} u_{1}\right)^{2}\right\rangle$, the dotted horizontal line, as given by Eq. (22). Here the mutual agreement is not very satisfactory. $r=3.6 \eta$ might still not be in the asymptotic range $r \rightarrow 0$.

ted the conditional averages with the beginning of a series in $v_{R}$,

$$
\left\langle\Delta_{r} u \mid v_{R}\right\rangle=f_{1}(r, R) v_{R}+f_{3}(r, R) v_{R}^{3} .
$$

No even power in $v_{R}$ is present, because the numerically obtained dependence of the conditional average on $v_{R}$ is clearly uneven. Inserting Eq. (28) into Eq. (24) leads to

$$
J_{n}(r, R)=f_{1}(r, R) S_{n}(R)+f_{3}(r, R) S_{n+2}(R) .
$$

If both $r$ and $R$ are in the ISR, the second term is small, see Fig. 6 and Table I, and the ISR factorization (7) obtained. If $r \in$ VSR the linear and linear plus cubic interpolations differ from each other and also from $J_{2}$ from the definition (2), see Figs. 8 and 9. Table I offers numbers for $f_{1}(r, R)$ and $f_{3}(r, R)$.

The series expansion of $J_{n}$ according to the numerical findings and to the odd power expansion of the conditional averages differs in its symmetry under reflection of the eddy velocity, $v(R) \rightarrow-v(R)$, from the VSR fusion rule (15). It behaves as $(-1)^{n}$, while Eq. (15) goes as $(-1)^{n+1}$. It thus is not yet clear, how the VSR rule $J_{n} \sim S_{n+1}(R) / R$ can be reconciled with the conditional average results. Further analysis is clearly necessary to get a sufficiently firm base for the $r$-VSR fusion properties.

If the flow field curvature $\Delta_{r} u$ is not discretized the resulting correlation function has some resemblance to 

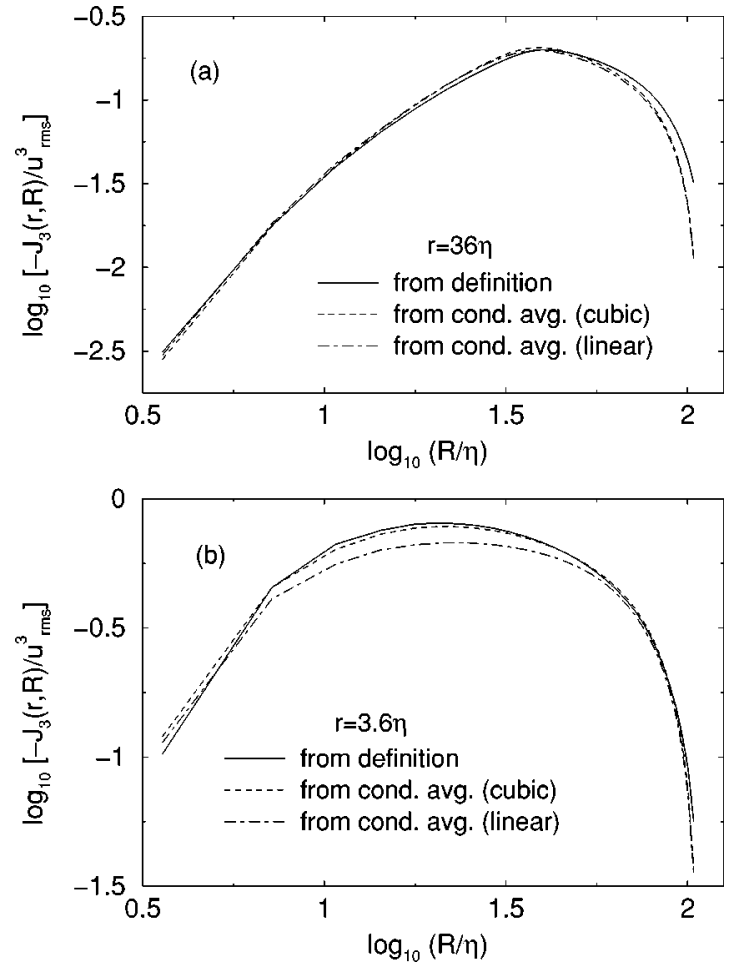

FIG. 9. $-J_{3}(r, R)$ versus $R / \eta$ for the two cases $r=36 \eta$ (a) and $r=3.6 \eta(\mathrm{b})$. With the cubic fit for the conditional average there is good agreement between the direct and the conditional average calculations of $J_{3}$. See also Fig. 8.

$\left\langle\epsilon v^{n-1}(R)\right\rangle$, where $\epsilon(\boldsymbol{x}, t)$ denotes the local dissipation rate per mass. In Ref. [2] so-called bridge relations for such objects have been advocated, saying here that

$$
\left\langle\epsilon v^{n-1}(R)\right\rangle \propto S_{n+2}(R) / R .
$$

This relation compares with the $r$-VSR factorization rule (15). But note, $\epsilon$ in Eq. (30) contains two factors of $u$ and is a (gradient $u$ )-squared and thus is a different correlation function as $J_{n}$. The bridge relation (30) has recently been checked [16] by numerical solution on a $512^{3}$ lattice, $\operatorname{Re}_{\lambda}$
TABLE I. Representative values for the coefficients $f_{1}(r, R)$ and $f_{3}(r, R)$ of the conditional average interpolation formula (28). Upper: the ISR case $r=36 \eta$; lower: the VSR choice $r=3.6 \eta$. The cubic term is markedly larger if $r$ is in the VSR.

\begin{tabular}{|c|c|c|c|c|}
\hline$R / \eta$ & & 36 & 54 & 72 \\
\hline$f_{1}$ & & 1.12 & 0.852 & 0.702 \\
\hline$f_{3} / f_{1}$ & & 0.00221 & 0.00184 & 0.00166 \\
\hline$R / \eta$ & 18 & 36 & 54 & 72 \\
\hline$f_{1}$ & 7.90 & 3.97 & 3.00 & 2.58 \\
\hline$f_{3} / f_{1}$ & 0.0167 & 0.00685 & 0.00306 & 0.00274 \\
\hline
\end{tabular}

$=220$ and 300 , and found to be valid within reasonable approximation in a compensated plot. It seems worthwhile to also study the conditional average representation which as we found may be more sensitive.

To conclude, the ISR factorization or "fusion" rules are shown to be "valid" under various different checks. But we also caution the reader: In Ref. [7] it has been pointed out that in order to have a very clear test of the fusion rule predictions, one has to have $\operatorname{Re}_{\lambda} \approx 2000$. There is no change in any numerical study to achieve this. However, the benefit of numerical studies can be good resolution towards small scales. We find that the small scale $r$-VSR fusion rules are far less clear. The corresponding conditional probabilities are objects to study these fusion rules more sensitively. The conditional average field curvature on scale $r, \Delta_{r} u$, essentially depends linearly on the conditioning eddy velocity fluctuation, irrespective of $r$ less than or beyond $\eta$.

It is our pleasure to acknowledge stimulating discussions with A. Fairhall, I. Procaccia, L. Biferale, and F. Toschi. This work was supported by the German-Israeli Foundation (GIF), and by the DFG through Grant No. SFB185-D3. It also is part of the research program of the Stichting voor Fundamenteel Onderzoek der Materie (FOM), which is financially supported by the Nederlandse Organisatie voor Wetenschappelijk Onderzoek (NWO). Computer time was supplied by the NIC in Jülich.
[1] A.L. Fairhall, V.S. L'vov, and I. Procaccia, Europhys. Lett. 43, 277 (1998).

[2] V.S. L'vov and I. Procaccia, Phys. Rev. Lett. 77, 3541 (1996).

[3] V.S. L'vov and I. Procaccia, Phys. Rev. Lett. 76, 2898 (1996).

[4] V.S. L'vov and I. Procaccia, Phys. Rev. E 54, 6268 (1996).

[5] S. Grossmann, D. Lohse, and A. Reeh, Phys. Rev. E 56, 5473 (1997)

[6] S. Grossmann, D. Lohse, and A. Reeh, Phys. Fluids 9, 3817 (1997)

[7] R. Benzi, L. Biferale, and F. Toschi, Phys. Rev. Lett. 80, 3244 (1998); R. Benzi, L. Biferale, G. Ruiz-Chavarria, S. Ciliberto, and F. Toschi, Phys. Fluids 11, 2215 (1999).

[8] A.N. Kolmogorov, J. Fluid Mech. 13, 82 (1962).
[9] A.M. Obukhov, J. Fluid Mech. 13, 77 (1962).

[10] A.L. Fairhall, B. Dhruva, V.S. L'vov, I. Procaccia, and K.R. Sreenivasan, Phys. Rev. Lett. 79, 3174 (1997).

[11] E.S.C. Ching, V.S. L'vov, E. Podivilov, and I. Procaccia, Phys. Rev. E 54, 6364 (1996).

[12] V. Yakhot and Y. Sinai, Phys. Rev. Lett. 63, 1965 (1991).

[13] P. Kailasnath, K.R. Sreenivasan, and J.R. Saylor, Phys. Fluids A 5, 3207 (1993).

[14] R.H. Kraichnan, Phys. Rev. Lett. 72, 1016 (1994).

[15] A.L. Fairhall, O. Gat, V.S. L'vov, and I. Procaccia, Phys. Rev. E 53, 3518 (1996).

[16] A. Ceani and D. Biskamp, Europhys. Lett. 46, 332 (1999). 